\title{
Effects of Primary Tumor Resection on Metastatic Breast Cancer Survival and the Predictive Power of Neutrophil: Lymphocyte Ratio on Prognosis
}

\author{
(1) Yaşar Çöpelci' ${ }^{1}$ (1) Umut Rıza Gündüz², (1) Bülent Dinç², (1) Nurhan Haluk Belen², (1) Şeyda Gündüz ${ }^{3}$ \\ ${ }^{1}$ Department of General Surgery, University of Health Sciences Turkey, Erzurum Regional Training and Research Hospital, Erzurum, Turkey \\ ${ }^{2}$ Department of General Surgery, University of Health Sciences Turkey, Antalya Training and Research Hospital, Antalya, Turkey \\ ${ }^{3}$ Clinic of Medical Oncology, Memorial Antalya Hospital, Antalya, Turkey
}

\begin{abstract}
Objective: The aim was to investigate the effect of primary tumor resection (PTR) on survival in metastatic breast cancer patients and to assess the power of the neutrophil-to-lymphocyte ratio (NLR) regarding the prediction of prognosis in this patient group.

Materials and Methods: Female patients diagnosed with and starting treatment for metastatic breast cancer from 2003 to 2016 in the general surgery and oncology clinics at a single center were retrospectively reviewed. Pre-treatment NLR value and survival situations were evaluated.

Results: A total of 117 patients were enrolled. The disease-specific survival (DSS) of the patients was 41.4 months. When stratified into PTR and systemic treatment $(\mathrm{ST})$ groups, there was no difference in the survival $(\mathrm{p}=0.054) ; 43.5$ months in the PTR group vs 30.7 months in the ST group. When hormone receptor (HR)-positive/human epidermal growth factor receptor 2 (HER2)-negative subgroups were analyzed, DSS was significantly longer $(p=0.02)$ in the PTR group (55.4 months) compared to the ST group (41.8 months). Finally, in patients with an NLR of $<2.3$, DSS was significantly longer ( $p=0.03$ ) in the PTR group (56.1 months) compared to the ST group (25.2 months).

Conclusion: These results suggest that DSS can be increased with PTR in selected patients with a diagnosis of metastatic breast cancer. NLR may be useful in selecting patients for appropraite treatment modality.
\end{abstract}

Keywords: Metastatic breast cancer, primary tumor resection, neutrophil-to-lymphocyte ratio, survival

Cite this article as: Çöpelci Y, Gündüz UR, Dinç B, Haluk Belen N, Gündüz Ş. Effects of Primary Tumor Resection on Metastatic Breast Cancer Surviva and the Predictive Power of Neutrophil: Lymphocyte Ratio on Prognosis. Eur J Breast Health 2021; 17(4): 322-327.

\section{Key Points}

- DSS was 43.5 months in the PTR group and 30.7 months in the ST group. Survival difference between the groups was not significant.

- In the HR-positive and HER2-negative subgroups, PTR was associated with longer DSS.

- In the NLR <2.3 subgroup, PTR was associated with longer DSS.

- $\quad$ PTR increased DSS rates in selected patient subgroups with metastatic breast cancer. NLR can be used as an effective tool in patient selection.

\section{Introduction}

Breast cancer is the most commonly diagnosed cancer in women and the most frequent reason for female cancer-related deaths in the world (1). In Turkey, the incidence of female breast cancer is 43.8/100,000 women, and 6\% of all patients diagnosed with breast cancer have been reported to have metastasis (2). Metastatic breast cancer is considered an incurable disease, and patients are usually provided palliative care. Nevertheless, advances in systemic treatment (ST) have significantly improved the control of metastatic diseases, thus offering prolonged survival. In this context, the role of primary tumor resection (PTR) in survival has, therefore, become a matter worth investigating. According to current practice, surgery is limited to symptomatic support in the treatment of metastatic breast cancer, but recent studies have suggested that survival and life quality can be increased in patients undergoing PTR and subsequently treated with an appropriate ST course $(3,4)$.

The search for new inflammatory markers for various diseases has been investigated for some time. Of particular interest is the physiological response of leucocytes to stress, a phenomenon that causes an increase in the neutrophil count and a concomitant relative decrease in lymphocytes. 
Based on this physiological mechanism, the neutrophil-to-lymphocyte ratio (NLR) has been proposed as a simple inflammatory marker (5). NLR has been studied as a biomarker in various tumor types and some studies have investigated the relationship between NLR and survival for metastatic breast cancer patients (6).

The aim of this study was to investigate the effect of PTR on survival in metastatic breast cancer patients and to assess the power of the NLR in terms of predicting prognosis in this patient group. In this way, it was hoped to demonstrate the effectiveness of the NLR in determining patients with metastatic breast cancer who would benefit most from primary tumor surgery.

\section{Materials and Methods}

After securing approval from the ethics committee, the records of all patients who were diagnosed with and started treatment for metastatic breast cancer from 2003 to 2016 in our center were retrospectively reviewed. All eligible patients had received no treatment prior to their admission. The patients were stratified into two groups: the PTR group, who underwent PTR followed by systemic treatment (ST), and the ST group, who only received ST.

Patient information was gathered from the hospital information management system (SARUS DBMS, EES Ltd. Şti, Ankara, Turkey) data hosted by clinical archives using File Maker Pro 7 (Claris International Inc., Santa Clara, CA, USA). Other data were obtained from the Death Declaration System (DDS) of the Turkish Ministry of Health (www.obs.saglik.gov.tr). The patients who died due to the disease and the dates of death were determined from the DDS. Patients who died for different reasons and those who underwent surgery for metastasis other than primary tumor were excluded from the study.

For all patients in the study, age at the time of diagnosis of stage IV breast cancer, gender, menopause status, time of initial diagnosis, histopathology results, positivity or not for estrogen receptor, progesterone receptor, and human epidermal growth factor receptor 2 (HER2), metastasis status, and time of complete blood count test at initial diagnosis data has been recorded.

The diagnosis of metastatic disease was made prior to treatment initiation by radiological, laboratory and pathological examinations, including ultrasound, positron emission tomography-computed tomography (PET CT), bone scintigraphy, tomography, and biopsy. Surgical procedures were performed for palliative purposes and were classified as follows: breast-conserving surgery, including resection of the primary tumor with tumor negative surgical margin; modified radical mastectomy; and simple mastectomy.

Systemic therapies were administered by patient-specific multidisciplinary decision. Disease-specific survival (DSS) was documented by calculating the time period from initial diagnosis to time of death, as recorded in the DDS, expressed as months. Data gathering was completed by November 2018, and the last follow up visit was chosen as the end-point for data collection in those patients who were still alive at the end of the data collection period.

For the other aim of the study, the complete blood count acquired prior to the initiation of treatment was evaluated. Neutrophil and lymphocyte counts and the NLR at first admission were also calculated for each patient.
Statistical analyses were performed using SPSS version 20 (IBM Inc., Armonk, NY, USA). An investigation of survival with univariate analyses was performed using the log rank test. Cox regression analysis was used via the retroactive selection method for investigating individual factors for survival prediction in multivariate analysis. Survival rates were calculated using the Kaplan-Meier method (Figures 1-3). The log rank test was used for evaluating the effect of the median value of the NLR on survival. Receiver operating (ROC) curves were generated, and the area under the curves was calculated to assess the extent to which changes in NLRs were capable of distinguishing 5-year disease-specific survival (DSS). Youden's index was utilized to determine the appropriate cut-off value for NLRs. Calculations with $<5 \%$ Type 1 error were accepted as statistically significant.

\section{Results}

A total of 117 female patients with metastatic breast cancer, with a median (range) age of 54 (26-86) years were enrolled. Among these patients, $38(32.5 \%)$ were premenopausal and $79(67.5 \%)$ were postmenopausal. DSS for the whole cohort was 41.4 months. Median age was 60 years in the ST group and 50 years in the PTR group. The primary tumor was surgically excised in 55 patients $(47 \%)$ and not treated surgically in 62 patients $(53 \%)$. When the metastatic areas of primary tumor were investigated, it was found that $52(44.4 \%)$ patients had bone metastasis only, 27 (23.1\%) had visceral metastasis, and $38(32.5 \%)$ had both bone and visceral metastasis (Table 1).

Overall survival (OS) durations were: 47.2 months in patients with bone metastasis only, 40.2 months in patients with visceral metastasis, and 23.8 months in patients with both bone and visceral metastasis. OS was significantly higher in patients who only had bone metastasis $(\mathrm{p}=0.032)$.

Cox regression analysis revealed a statistically significant relationship between DSS and hormone receptor (HR) status and the NLR $(\mathrm{p}<0.05)$, whereas there were no relations with age $(\mathrm{p}=0.86)$, menopause status $(\mathrm{p}=0.77)$, surgery status $(\mathrm{p}=0.15)$, or metastatic status ( $\mathrm{p}=0.22$ ). Following multivariate analysis, the NLR and HR status continued to exhibit a statistically significant relation with DSS ( $\mathrm{p}=0.03$ and $\mathrm{p}=0.02$, respectively).

Survival was 43.5 months in the PTR group and 30.7 months in the ST group. DSS was not significantly different between the two groups $(\mathrm{p}=0.054)$.

There were 64 patients with HR-positive/HER2-negative metastasis and the DSS was 55.4 months in the PTR group $(\mathrm{n}=27)$ and 41.8 months in the ST group $(\mathrm{n}=37)$, which was statistically significant $(p=0.02)$. When the NLR was evaluated for the whole cohort, the median value was found to be 2.3. In patients with an NLR of $<2.3$, survival was 56.1 months in the PTR group and 25.2 months in the ST group. This survival difference between the two groups was statistically significant in favor of the PTR group $(\mathrm{p}=0.03)$ (Table 2).

\section{Discussion and Conclusion}

This study demonstrated that PTR followed by ST may provide better DSS compared to ST in patients with metastatic breast cancer, but only in selected patients. Although survival in the PTR group was longer than in the ST group, no statistically significant difference was found between the two groups in terms of DSS. However, subgroup analysis revealed that PTR had a positive effect on survival in patients 
with HR-positive/HER2-negative metastasis. Furthermore, in patients with an NLR of $<2.3$, survival of the PTR group was longer than in the ST group ( $\mathrm{p}=0.03)$.

Since Rapiti et al. (7) published the results of an important study in 2006 that reported that PTR reduced cancer-related deaths in patients with metastatic breast cancer, this procedure has been of interest to clinicians managing this patient group. Since 2006, many retrospective and a few prospective studies have been conducted. However, PTR in metastatic breast cancer patients remains controversial.

In a recently published, comprehensive meta-analysis, the effectiveness of locoregional therapy (LRT) in patients with de novo stage IV breast cancer was investigated. Meta-analysis results from 216,066 patients revealed that LRT can reduce mortality by $31.8 \%$. Furthermore, it has been reported that surgery can specifically reduce mortality by $36.2 \%$ (8). Studies comparing OS results also reported that PTR is associated with longer OS (9-11).

In the retrospective study conducted by Babiera et al. (12), although single-site metastasis and HER2/Neu gene mutation were negative, they found no statistically significant difference in survival, although a longer survival trend was reported. That study further showed that PTR was associated with increased metastasis-progression-free survival. Meanwhile, in a study on the relationship between metastasis and survival, Fields et al. (13) reported that PTR in metastatic breast cancer patients reduced the incidence of death, not only in patients with bone metastases but also in those with metastases at other sites. In our study, we found no relation between metastatic site and DSS but this result may be due to the effect of the sample size or the classification of metastasis status.

Table 1. Demographic data

\begin{tabular}{|c|c|c|c|c|}
\hline & & PTR Group & ST Group & Total \\
\hline Age & & 50 & 60 & 54 \\
\hline \multirow{2}{*}{ Menopause status } & Premenopausal & $21(18 \%)$ & $17(14.5 \%)$ & $38(32.5 \%)$ \\
\hline & Postmenopausal & $34(29 \%)$ & $45(38.5 \%)$ & $79(67.5 \%)$ \\
\hline \multirow{2}{*}{ Hormone receptor status } & $\mathrm{HR}+$ & $35(30 \%)$ & $51(43.5 \%)$ & $86(73.5 \%)$ \\
\hline & $\mathrm{HR}-$ & $20(17 \%)$ & $11(9.5 \%)$ & $31(26.5 \%)$ \\
\hline \multirow{12}{*}{ HER2 receptor status } & HER2+ & $14(12 \%)$ & $18(15.3 \%)$ & $32(27.3 \%)$ \\
\hline & HER2- & $41(35 \%)$ & $44(37.7 \%)$ & $85(72.7 \%)$ \\
\hline & Bone-only & $25(21.4 \%)$ & $27(23.1 \%)$ & $52(44.5 \%)$ \\
\hline & Visceral only & 17 (14.5\%) & $10(8.5 \%)$ & $27(23 \%)$ \\
\hline & Lung & 9 & 4 & 13 \\
\hline & Liver & 4 & 5 & 9 \\
\hline & Lung, liver & 0 & 1 & 1 \\
\hline & Mediastinal & 4 & 0 & 4 \\
\hline & Bone and visceral & $13(11.1 \%)$ & $25(21.4 \%)$ & $38(32.5 \%)$ \\
\hline & Lung, bone & 1 & 12 & 13 \\
\hline & Lung, brain, bone & 0 & 1 & 1 \\
\hline & Lung, liver, bone & 2 & 4 & 6 \\
\hline \multirow[t]{6}{*}{ Metastasis sites } & Lung, pancreas, bone & 1 & 0 & 1 \\
\hline & Brain, bone & 1 & 2 & 3 \\
\hline & Brain, liver, bone & 0 & 1 & 1 \\
\hline & Liver, bone & 8 & 4 & 12 \\
\hline & Liver, mediastinal, bone & 0 & 1 & 1 \\
\hline & MRM & $46(39.3 \%)$ & - & $55(47 \%)$ \\
\hline \multirow{4}{*}{ Type of surgery } & Simple mastectomy & $7(6 \%)$ & - & 55 \\
\hline & $\mathrm{BCS}$ & $2(1.7 \%)$ & - & 55 \\
\hline & Chemotherapy & 40 (34.2\%) & $43(36.8 \%)$ & $83(71 \%)$ \\
\hline & Endocrine therapy & $6(5.2 \%)$ & $5(4.3 \%)$ & $11(9.5 \%)$ \\
\hline \multirow[t]{2}{*}{ Systemic therapies } & $\begin{array}{l}\text { Chemotherapy plus endocrine } \\
\text { therapy }\end{array}$ & $2(1.7 \%)$ & $1(0.8 \%)$ & $3(2.5 \%)$ \\
\hline & Missing & $7(6 \%)$ & $13(11 \%)$ & $20(17 \%)$ \\
\hline
\end{tabular}


A prospective randomized study by Badwe et al. (14) compared PTR with ST in the treatment of metastatic breast cancer patients and examined OS using a sample of 350 metastatic breast cancer patients between 2005 and 2013. They reported no difference in OS between the PTR and ST groups. Moreover, through subgroup analyses, they found that menopause, metastatic areas, estrogen and progesterone receptor or HER2 status did not make a significant difference to OS. However, there are important factors affecting the results in this study. The patients, in contrast to our study, were metastatic breast cancer patients who had previously undergone chemotherapy and responded to treatment before being included in the study and then randomized. Furthermore, although 107 (31\%) of the 350 patients in their study had HER2-positive disease, only $8 \%$ of these patients were able to receive HER2-targeted therapy due to financial constraints (14). The presence of these factors may have had a significant impact on treatment efficacy and survival, and this should be kept in mind when comparing survival outcomes.

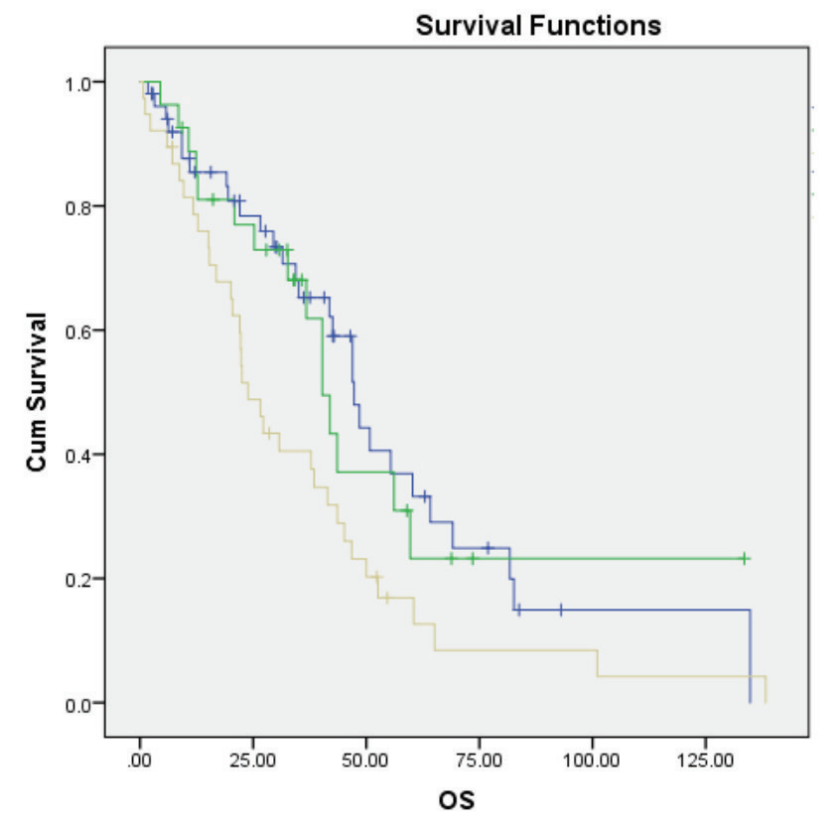

Metastases sites and mortality: —Bone — Visceral — Bone and visceral

+ Bone censored + Visceral censored

+ Bone and visceral censored

Figure 1. Survival difference between metastatic sites

OS: Overall survival; Cum: Cummulative

Table 2. Statistically significant parameters

\begin{tabular}{lccc|} 
& PTR & ST & P \\
\hline $\begin{array}{l}\text { NLR }<2.3 \\
(\mathbf{n}=\mathbf{4 9})\end{array}$ & 26 & 23 & \\
DSS (month) & 56.1 & 25.2 & $\mathbf{0 . 0 3}$ \\
ER/PR (+), HER2 (-) $(\mathbf{n}=\mathbf{6 4})$ & 27 & 37 & \\
DSS (month) & 55.4 & 41.8 & $\mathbf{0 . 0 2}$ \\
\hline
\end{tabular}

Significant p-values are shown in bold and italic.

PTR: Primary tumor resection; ST: Systemic treatment; DSS: Diseasespecific survival; NLR: Neutrophil-to-lymphocyte; ER: Estrogen receptor; PR: Progesterone receptor; HER2: Human epidermal growth factor receptor 2; n: Number
The other prospective study, the MF07-01 trial, was designed by Soran et al. (15). This was a multicenter, phase III, randomized controlled study whose results were first published in 2016 and then in 2018 after a 5-year follow-up (16). A total of 274 metastatic breast cancer patients were randomized into two groups: one received ST after LRT and the other received ST alone, after which they were evaluated in terms of OS. Patients did not receive any treatment before being included in the study. The 5-year follow-up results revealed that $41.6 \%$ of the LRT group and $24.2 \%$ of the ST group were alive. In the LRT group, the risk of death was 34\% lower than that in the ST group. In subgroup analysis, OS was significantly higher in patients with HR-positive/

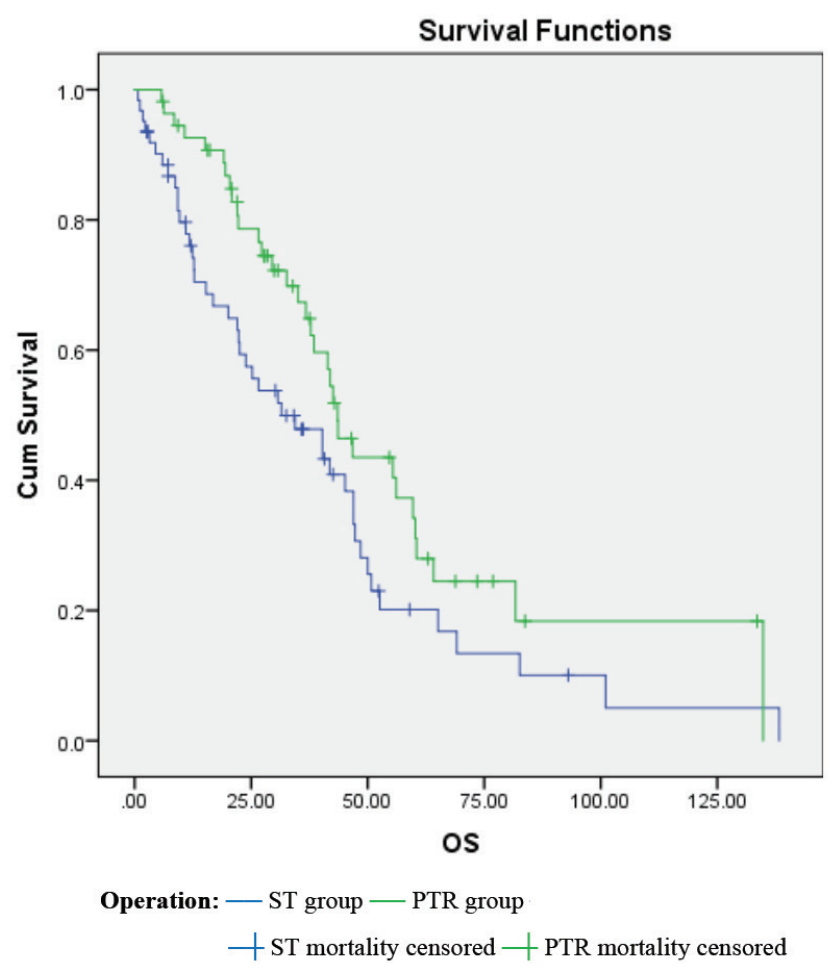

Figure 2. Survival difference between study groups

OS: Overall survival; Cum: Cummulative

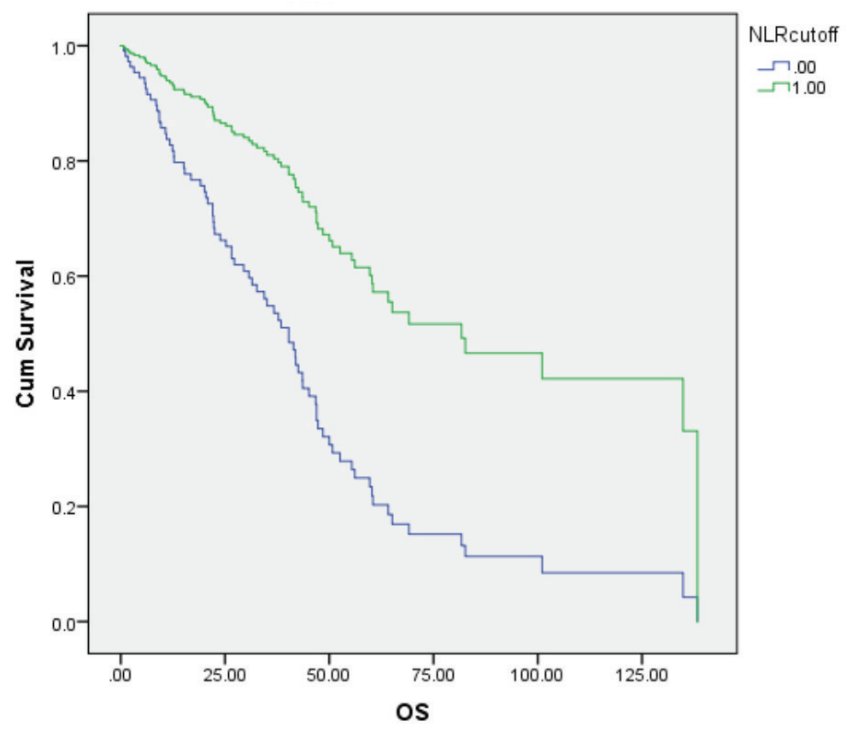

Figure 3. Survival difference between NLR cut-off values

OS: Overall survival; Cum: Cummulative; NLR: Neutrophil-to-lymphocyte 
HER2/Neu-negative, bone-only metastasis who were under 55 years of age in the LRT group compared to the ST group. Patients in the LRT group with bone-only metastasis had a survival outcome that was 14 months longer than that in the ST group (15).

To the best of our knowledge, our study is the first to evaluate the effect of PTR on survival and then examine the predictive significance of the NLR in metastatic breast cancer patients. In the NLR $<2.3$ subgroup, DSS was significantly longer in the PTR group compared with the ST group. Many studies have shown that NLR can be of prognostic value in breast cancer, similar to other cancers. However, most studies on NLR and its value in breast cancer did not specifically examine metastatic breast cancer.

Our study differs from the literature in that it specifically examines the prognostic value of NLR in metastatic breast cancer patients. A systematic review and meta-analysis by Ethier et al. (16) reported that a high NLR has been shown to have a negative effect on OS and disease-free survival, and has been identified as an easily accessible prognostic marker. It has also been reported that neutrophils inhibit the immune system and suppress the activity of lymphocytes and the T-cell response; thus, tumor growth may increase. Furthermore, high NLR can be considered an indicator of increased inflammation, which may also result in immunosuppessive effects and lymphocyte inhibition (16). Another report stated that NLR may be used as an independent prognostic factor for OS in metastatic breast cancer patients (17). Takuwa et al. (18) retrospectively examined the results of 171 metastatic breast cancer patients and showed a strong association between a high NLR and poor prognosis. Similarly, in a meta-analysis of 18 studies, Liu et al. (19) investigated the prognostic value of the NLR before breast cancer treatment and showed a correlation between a high NLR and poor prognosis in breast cancer patients.

A recent retrospective study by Iimori et al. (20) of 34 stage IV breast cancer patients undergoing endocrine therapy showed that a low NLR was associated with a reduction in treatment failure rates, progressionfree survival and an increase in OS. Multivariate analysis results showed that treatment response and a low NLR were independent factors for a better prognosis, suggesting that the NLR can be used as a predictive marker of endocrine treatment response in stage IV breast cancer patients (20). In the observational studies of Azad et al. (21), published in 2012, all stages of breast cancer in patients diagnosed and treated between 2004 and 2006 were evaluated for NLR. They found that those with an NLR of $>3.3$ had the the highest first- and fifth-year mortality rates, whereas those with an NLR of $<1.8$ had the lowest mortality rate. Thus, an NLR of $>3.3$ was shown to be an independent predictor of mortality (21). In our study, performing PTR in patients with NLR values below 2.3 significantly increased OS.

Our study has some limitations that should be noted. Primarily, this work was a retrospective, single-center study. Thus, it was subject to various limitations encountered in retrospective studies, such as the lack of regular records of adjuvant therapy regimens, which explains why the chosen surgical method was selected. The importance of PTR in metastatic breast cancer can be understood more clearly with the recent widespread, multicenter, prospective studies that continue to collect data. It was important in our study to have an equal number of study groups over a long period of time in order to follow multidisciplinary treatments and investigate the effect of us to determine the efficacy of using NLR in conducting appropriate patient selection and, subsequently, to recommend this practice.

In conclusion, the results of our study using ST following PTR in the treatment of metastatic breast cancer patients significantly increased survival in HR-positive/HER2-negative patients compared to ST only, with longer survival in patients with an NLR of $<2.3$, thus contributing to the literature on treatments for these patients. We believe that PTR may be an important treatment option in metastatic breast cancer patients and that NLR, as an indicator of systemic inflammation, can be a useful criterion in the selection and delivery of optimal therapy. However, in patients with stage IV breast cancer, further research is needed to evaluate the effect of patient selection on survival after PTR.

Ethics Committee Approval: This study was approved by the Ethics Committee of the University of Health Sciences Turkey, Antalya Training and Research Hospital with the decision number 2/007 dated 24/01/2019.

Informed Consent: Retrospective study.

Peer-review: Externally peer-reviewed

\section{Authorship Contributions}

Conception: Y.Ç., U.R.G., B.D., N.H.B., Ş.G.; Design: Y.Ç., U.R.G., B.D., N.H.B., Ş.G.; Data Collection and/or Processing: Y.Ç., U.R.G., B.D., N.H.B., Ş.G.; Analysis and/or Interpretation: Y.Ç., U.R.G., B.D., N.H.B., Ş.G.; Writing: Y.Ç., U.R.G., B.D., N.H.B., Ş.G.

Conflict of Interest: The authors declare no conflict of interest.

Financial Disclosure: The research has no financial grants and supports.

\section{References}

1. Bray F, Ferlay J, Soerjomataram I, Siegel RL, Torre LA, Jemal A. Global cancer statistics 2018: GLOBOCAN estimates of incidence and mortality worldwide for 36 cancers in 185 countries. CA Cancer J Clin 2018; 68: 394-424. (PMID: 30207593) [Crossref]

2. Republic of Turkey Ministry of Health. Health Statistics Yearbook 2017. Available at: https://dosyasb.saglik.gov.tr/Eklenti/30148,ingilizcesiydijiv1 pdf.pdf?0 [Crossref]

3. Lee JS, Toktas $\mathrm{O}$, Soran A. Role of locoregional treatment in de novo stage IV breast cancer. Clin Med Insights Oncol 2020; 14: 1179554920942440. (PMID: 32994701) [Crossref]

4. Teshome M. Role of operative management in stage IV breast cancer. Surg Clin North Am 2018; 98: 859-868. (PMID: 30005779) [Crossref]

5. Faria SS, Fernandes PC, Silva MJB, Lima VC, Fontes W, FreitasJunior R, et al. The neutrophil-to-lymphocyte ratio: a narrative review. Ecancermedicalscience 2016; 10: 702. (PMID: 28105073) [Crossref]

6. Guthrie GJ, Charles KA, Roxburgh CS, Horgan PG, McMillan DC, Clarke SJ. The systemic inflammation-based neutrophil-lymphocyte ratio: experience in patients with cancer. Crit Rev Oncol Hematol 2013; 88: 218-230. (PMID: 23602134) [Crossref]

7. Rapiti E, Verkooijen HM, Vlastos G, Fioretta G, Neyroud-Caspar I, Sappino AP, et al. Complete excision of primary breast tumor improves survival of patients with metastatic breast cancer at diagnosis. J Clin Oncol 2006; 24: 2743-2749. (PMID: 16702580) [Crossref]

8. Lane WO, Thomas SM, Blitzblau RC, Plichta JK, Rosenberger LH, Fayanju OM, et. al. Surgical resection of the primary tumor in women with de novo stage IV breast cancer. Ann Surg 2019; 269: 537-544. (PMID: 29227346) [Crossref] 


\section{Çöpelci et al. Primary Tumor Resection in Metastatic BC}

9. Lang JE, Tereffe W, Mitchell MP, Rao R, Feng L, Meric-Bernstam F, et al. Primary tumor extirpation in breast cancer patients who present with stage IV disease is associated with improved survival. Ann Surg Oncol 2013; 20: 1893-1899. (PMID: 23306905) [Crossref]

10. Blanchard DK, Shetty PB, Hilsenbeck SG, Elledge RM. Association of surgery with improved survival in stage IV breast cancer patients. Ann Surg 2008; 247: 732-738. (PMID: 18438108) [Crossref]

11. Ruiterkamp J, Voogd AC, Tjan-Heijnen VC, Bosscha K, Linden YM, Rutgers EJT, et al. SUBMIT: Systemic therapy with or without up front surgery of the primary tumor in breast cancer patients with distant metastases at initial presentation. BMC Surg 2012; 12: 5. (PMID: 22469291) [Crossref]

12. Babiera GV, Rao R, Feng L,Meric-Bernstam F, Kuerer HM, Singletary $\mathrm{SE}$, et al. Effect of primary tumor extirpation in breast cancer patients who present with stage IV disease and an intact primary tumor. Ann Surg Oncol 2006; 13: 776-782. (PMID: 16614878) [Crossref]

13. Fields RC, Jeffe DB, Trinkaus K, Zhang Q, Arthur C, Aft R, et al. Surgical resection of the primary tumor is associated with increased long-term survival in patients with stage IV breast cancer after controlling for site of metastasis. Ann Surg Oncol 2007; 14: 3345-3351. (PMID: 17687611) [Crossref]

14. Badwe R, Hawaldar R, Nair N, Kaushik R, Parmar V, Siddique S, et al. Locoregional treatment versus no treatment of the primary tumour in metastatic breast cancer: an open-label randomised controlled trial. Lancet Oncol 2015; 16: 1380-1388. (PMID: 26363985) [Crossref]

15. Soran A, Ozmen V, Ozbas S, Karanlik H, Muslumanoglu M, Canturk NZ, et al. Randomized trial comparing resection of primary tumor with no surgery in stage iv breast cancer at presentation: protocol MF07-01. Ann Surg Oncol 2018; 25: 3141-3149. (PMID: 29777404) [Crossref]

16. Ethier JL, Desautels D, Templeton A, Shah PS, Amir E. Prognostic role of neutrophil-to-lymphocyte ratio in breast cancer: a systematic review and meta-analysis. Breast Cancer Res 2017;19:2. (PMID: 28057046) [Crossref]

17. Corbeau I, Jacot W, GuiuS. Neutrophil to lymhocyte ratio as prognostic and predictive factor in breast cancer patients: a systematic review. Cancers (Basel) 2020; 12: 958. (PMID: 32295078) [Crossref]

18. Takuwa H, Tsuji W, Yamamoto Y, Shintaku M, Yotsumoto F. Low neutrophil-lymphocyte ratio correlates with extended survival in patients with metastatic breast cancer who achieved clinically complete response following multidisciplinary therapy: a retrospective study. Oncol Lett 2018; 15: 6681-6687. (PMID: 29725410) [Crossref]

19. Liu X, Qu J-K, Zhang J, Yan Y, Zhao X-X, Wang J-Z, et al. Prognostic role of pretreatment neutrophil to lymphocyte ratio in breast cancer patients: a meta-analysis. Medicine (Baltimore) 2017; 96 :e8101. (PMID: 29137007) [Crossref]

20. Iimori N, Kashiwagi S, Asano Y, Goto W, Takada K, Takahashi K, et al. Clinical significance of the neutrophil-to-lymphocyte ratio in endocrine therapy for stage iv breast cancer. In Vivo 2018; 32: 669-675. (PMID: 29695577) [Crossref]

21. Azab B, Bhatt VR, Phookan J, Murukutla S, Kohn N, Terjanian T, et al. Usefulness of the neutrophil-to-lymphocyte ratio in predicting short- and long-term mortality in breast cancer patients. Ann Surg Oncol 2012; 19 : 217-224. (PMID: 21638095) [Crossref] 